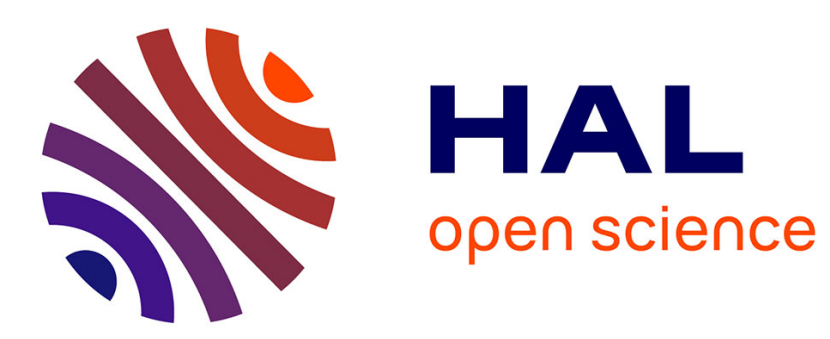

\title{
A nonlinear approach to the control of a disc-shaped aircraft
}

\author{
Jean-Marie Kai, Tarek Hamel, Claude Samson
}

\section{To cite this version:}

Jean-Marie Kai, Tarek Hamel, Claude Samson. A nonlinear approach to the control of a disc-shaped aircraft. CDC 2017 - 56th IEEE Conference on Decision and Control, Dec 2017, Melbourne, Australia. pp.2750-2755, 10.1109/CDC.2017.8264058 . hal-01629150

\section{HAL Id: hal-01629150 \\ https://hal.science/hal-01629150}

Submitted on 6 Nov 2017

HAL is a multi-disciplinary open access archive for the deposit and dissemination of scientific research documents, whether they are published or not. The documents may come from teaching and research institutions in France or abroad, or from public or private research centers.
L'archive ouverte pluridisciplinaire HAL, est destinée au dépôt et à la diffusion de documents scientifiques de niveau recherche, publiés ou non, émanant des établissements d'enseignement et de recherche français ou étrangers, des laboratoires publics ou privés. 


\title{
A nonlinear approach to the control of a disc-shaped aircraft
}

\author{
Jean-Marie Kai, Tarek Hamel and Claude Samson
}

\begin{abstract}
This paper describes a new control approach for scale-model airplanes. The proposed control solution is primarily geared towards drones whose lift surfaces can be approximated by a disc-shaped wing. Designed and analysed on the basis of a specific model of aerodynamic forces acting on the aircraft, it departs from other solutions in its capacity to handle important and rapidly changing attack angles within a large flight envelope. Simulation results illustrate its robust performance.
\end{abstract}

\section{INTRODUCTION}

Automatic control of aerial vehicles has been an active research area for decades. Solutions have been steadily maturing throughout the years leading to sophisticated autopilot systems for manned vehicles and fully autonomous flight and navigation systems for a range of military and civil applications. Nowadays, the emergence of modern embedded computing, sophisticated GPS positioning, the availability of low-cost MEMS sensor systems and reliable miniature pitot-tubes, along with a growing hobby market in low-cost light-weight remote controlled aerial vehicles, have opened a vast range of new civil and military applications. While the commercial landscape of aerial vehicles is characterised by a plethora of small start-up companies marketing different specialized platforms for specific applications, the trend is to equip these platforms with always more versatile and robust control systems.

A known limitation of commonly available control systems is their reliance on linear control techniques (LQ methods, $H_{\infty}, \mu$-synthesis,...) based on the linearization of the vehicle's dynamics about a small set of so-called trim trajectories (see [1], for instance). As long as the attack and sideslip angles, and their variations, are small these techniques are relevant. They are also well justified in the case of airliners that have to comply with drastic trajectory requirements in relation to passenger comfort and weather conditions. However, for highly manoeuvrable fighter aircraft and particularly sensitive-to-wind scale-model aircraft, trajectories can be much more aggressive and involve large and rapidly varying attack angles. Linear control techniques are then not best adapted. Several nonlinear feedback methods have been proposed over the past two decades to cover extended flight envelopes with guaranteed stability. The control architecture is more or less the same for all kind of aerial vehicles. It essentially consists in solving a hierarchical control problem: 1) guidance (also termed outer-loop) that

J.M. Kai and T. Hamel are with I3S, Université Côte d'Azur, CNRS, Sophia Antipolis, France, kai(thamel)@i3s.unice.fr.

C. Samson (corresponding author) is with INRIA and I3S UCACNRS, Sophia Antipolis, France, claude.samson@inria.fr, csamson@i3s.unice.fr. involves intermediary control variables calculated to asymptotically stabilize the vehicle's desired path (or trajectory), and 2) flight-control (also termed inner-loop) that calculates low-level control inputs (control surfaces angles, for instance, in the case of airplanes) ensuring the stabilization of the intermediary variables at their desired values determined at the guidance level. This type of control architecture relies on a time scale separation between the guidance part (slow dynamics) and the more reactive flight-control part (fast dynamics). While thrust vectoring (i.e. thrust direction and magnitude) is often chosen as guidance control input in the case of low-velocity VTOL vehicles with reduced lift surfaces [2], air-velocity vector (magnitude and direction) and bank angle are commonly used as guidance control inputs in the case of cruising aircraft endowed with important lift surfaces (airplanes, in particular). Taking the dynamics of aerodynamical forces into account in the control problem significantly complicates the design of flight-control systems. This problem has been addressed with various methods. Let us cite, for instance, linear control techniques combined with gain scheduling [3], LPV (Linear Parameter Varying) control [4], [5], and feedback linearization combined with LQ control [6] or robust stochastic nonlinear control [7]. The approach that we propose is different.

The objective of this work is to derive a generic control approach that can serve as a basis to design automatic control (autopilot) systems for a large class of scale model aircraft and aeroplane-like drones. The proposed control design methodology is inspired from our previous work [8] on the control of scale-model airplanes, itself an extension of our earlier works on VTOL drones and axisymmetric vehicles [9], but departs from it in the modelling of the aerodynamic forces applied to the vehicle and the way this model is used to asymptotically stabilize the sideslip angle at zero (balanced flight). While in [8] the control of this angle is decoupled from the control of the thrust direction, the orientation control problem is here termed as the problem of asymptotically stabilizing the complete vehicle's orientation at a desired orientation, itself calculated to ensure exponential stability of zero tracking errors. To satisfy our aim of generic applicability we have opted for a model of the aerodynamic forces acting on the vehicle that is both representative of the physics underlying the creation of the environmental forces and sufficiently simple to lend itself to control design and analysis. More precisely, the compromise here proposed consists in i) modelling an aircraft as a disc-shaped wing whose surface is approximately the sum of the main lift surfaces of a physical aircraft and ii) using a nonlinear model of the aerodynamic forces acting on this wing that, despite 
its simplicity and imperfection, is coherent with other models used in the literature dedicated to flight control. We make it clear that this model is used exclusively for control design and analysis purposes and that it is by no means viewed by the authors of the present article as an exact representation of a physical aircraft. Design and validation of a control law on this model is only the first stage of the specification of a complete autopilot system dedicated to a specific aircraft. For instance, a second stage will typically consist in bringing adjustments to the control law (parameter estimation, control gains calculations, design of low-level control surfaces, etc.) and in testing it on more sophisticated simulation models to evaluate its performance and robustness. It is only when it passes successfully all simulation tests that it can be implemented and tested on a real aircraft.

The paper is organized as follows. Section II recalls the equations of motion of an aircraft and presents a model for the aerodynamic forces acting on an aircraft whose shape is approximated by a disc. Section III first defines a set of admissible reference trajectories, encompassing trim trajectories, that can be exponentially stabilized with the proposed control approach. Next, taking the aircraft propelling thrust and angular velocity as intermediary control inputs, the design of a local exponential stabilizer is presented. Simulation results involving a challenging reference trajectory, large initial tracking errors and several modelling errors purposely introduced to test the the control robustness, are reported in Section IV to illustrate the control performance. Concluding remarks and a short list of possible extensions of this work are given in the last Section $\mathrm{V}$.

\section{CONTROL MODEL}

\section{A. Notation}

Throughout the paper, $\boldsymbol{E}^{3}$ denotes the $3 D$ Euclidean vector space and vectors in $\boldsymbol{E}^{3}$ are denoted with bold letters. Inner and cross products in $\boldsymbol{E}^{3}$ are denoted by the symbols - and $\times$ respectively. The following notation is used.

- $G$ denotes the body's center of mass (CoM);

- $\mathcal{I}=\left\{O ; \boldsymbol{\imath}_{0}, \boldsymbol{\jmath}_{0}, \boldsymbol{k}_{0}\right\}$ is an inertial frame;

- $\mathcal{B}=\{G ; \boldsymbol{\imath}, \boldsymbol{\jmath}, \boldsymbol{k}\}$ is a body-fixed frame;

- $\boldsymbol{\omega}$ is the angular velocity of $\mathcal{B}$ w.r.t. $\mathcal{I}$, i.e.

$$
\frac{d}{d t}(\boldsymbol{i}, \boldsymbol{j}, \boldsymbol{k})=\boldsymbol{\omega} \times(\boldsymbol{i}, \boldsymbol{j}, \boldsymbol{k})
$$

- $m$ is the body mass;

- $\boldsymbol{v}$ is the CoM's velocity w.r.t. the inertial frame;

- $\boldsymbol{a}$ is the CoM's acceleration w.r.t. the inertial frame;

- $\boldsymbol{g}=g_{0} \boldsymbol{k}_{0}$ is the gravitational acceleration;

- $\boldsymbol{v}_{w}$ is the ambient wind velocity w.r.t. $\mathcal{I}$;

- $\boldsymbol{v}_{a}=\boldsymbol{v}-\boldsymbol{v}_{w}$ is the body air-velocity. The direction of $\boldsymbol{v}_{a}$ in body frame can be characterized by two angles $\alpha$ and $\beta$ such that

$$
\boldsymbol{v}_{a}=\left|\boldsymbol{v}_{a}\right|(\cos \alpha(\cos \beta \boldsymbol{i}+\sin \beta \boldsymbol{\jmath})+\sin \alpha \boldsymbol{k})
$$

- The coordinate vector of any $\boldsymbol{\xi} \in \boldsymbol{E}^{3}$ w.r.t. the bodyfixed frame $\mathcal{B}$ is denoted by the ordinary letter $\xi$, i.e. $\boldsymbol{\xi}=\xi_{1} \boldsymbol{\imath}+\xi_{2} \boldsymbol{\jmath}+\xi_{3} \boldsymbol{k}$ with $\xi=\left[\xi_{1}, \xi_{2}, \xi_{3}\right]^{\top}$.
In the case of an airplane, and with a proper choice of the body frame unit vectors, $\alpha=\arcsin \left(v_{a, 3} /\left|\boldsymbol{v}_{a}\right|\right)$ and $\beta=\arctan \left(v_{a, 2} / v_{a, 1}\right)$ denote the attack angle and sideslip angle respectively. This definition slightly differs from the one classically used in aeronautics but the two definitions are locally equivalent for small angles. Note also that, like any parametrization of the unit sphere by two angles, this representation is not uniquely defined everywhere. More precisely $\beta$ cannot be defined by continuity at $\alpha= \pm \pi / 2$.

\section{B. Aerodynamic forces}

The resultant aerodynamic force $\boldsymbol{F}_{a}$ applied to a rigid body moving with air-velocity $\boldsymbol{v}_{a}$ is traditionally decomposed into the sum of a drag force $\boldsymbol{F}_{D}$ along the direction of $\boldsymbol{v}_{a}$ and a lift force $\boldsymbol{F}_{L}$ perpendicular to this direction, i.e.

$$
\boldsymbol{F}_{a}=\boldsymbol{F}_{D}+\boldsymbol{F}_{L}
$$

The intensities of drag and lift forces are essentially proportional to $\left|\boldsymbol{v}_{a}\right|^{2}$ modulo variations characterized by two dimensionless functions $C_{D}$ and $C_{L}$, which depend in the first place on the orientation of $\boldsymbol{v}_{a}$ w.r.t. the body, but also on the Reynolds number $R_{e}$ and Mach number $M$. These dimensionless functions are called the aerodynamic characteristics of the body, or drag coefficient and lift coefficient respectively. More precisely

$$
\boldsymbol{F}_{D}=-\eta_{a}\left|v_{a}\right| C_{D} \boldsymbol{v}_{a}, \quad \boldsymbol{F}_{L}=\eta_{a}\left|v_{a}\right| C_{L} \boldsymbol{v}_{a}^{\perp}
$$

with

- $\boldsymbol{v}_{a}^{\perp}$ some vector perpendicular to $\boldsymbol{v}_{a}$ and such that $\left|\boldsymbol{v}_{a}^{\perp}\right|=\left|v_{a}\right|$

- $\eta_{a}:=\frac{\rho \Sigma}{2}$ with $\rho$ the free stream air density, and $\Sigma$ an area germane to the body shape.

Throughout the paper, we assume that the resultant aerodynamic force $\boldsymbol{F}_{a}$ applies at $G$ so that it does not produce a torque. More exactly, we assume that the torque produced by $\boldsymbol{F}_{a}$ can, in practice, be overcome by a chosen control torque. A common assumption in the flight control literature, when $\alpha, \beta$ are well defined, is that the aerodynamic characteristics depend essentially on $\alpha$ and $\beta$ (i.e. $C_{D}=C_{D}(\alpha, \beta)$ and $\left.C_{L}=C_{L}(\alpha, \beta)\right)$. Accordingly, we neglect here the dependence of the aerodynamic characteristics on the Reynolds and Mach numbers.

\section{Body dynamics equations}

We assume that the control inputs consist of a thrust force $\boldsymbol{T}=T \boldsymbol{\imath}$ applied at $G$, with $T$ the thrust intensity, and a torque vector $\boldsymbol{\Gamma}_{G}$. The body dynamics equations are then given by (1) complemented with the classical Newton-Euler equations:

$$
\begin{aligned}
& m \boldsymbol{a}=m \boldsymbol{g}+\boldsymbol{F}_{a}+T \boldsymbol{i} \\
& J \dot{\omega}=-S(\omega) J \omega+\Gamma_{G}
\end{aligned}
$$

with $J$ the body inertia matrix, and $\Gamma_{G}$ the vector of coordinates of $\boldsymbol{\Gamma}_{G}$ in the body frame. In view of Eq. (6), $\omega$ can be modified at will via the choice of the control torque $\Gamma_{G}$ so that one can consider the angular velocity $\omega$ 
as an intermediate control input. The corresponding physical assumption is that "almost" any desired angular velocity can be obtained after a short transient time. This is a standard "backstepping" assumption. Once it is made, the vehicle's actuation consists in four input variables, namely, the thrust intensity $T$ and the three components of $\omega$. This assumption allows one to "eliminate" or, more precisely, postpone the complementary issue of producing a desired angular velocity via a torque $\Gamma_{G}$ that can be produced via various and specific physical means (control surfaces, in the case of a standard airplane). The body dynamics equations are then reduced to (1) and (5).

\section{Modelling of aerodynamic forces on a disc-shaped wing}

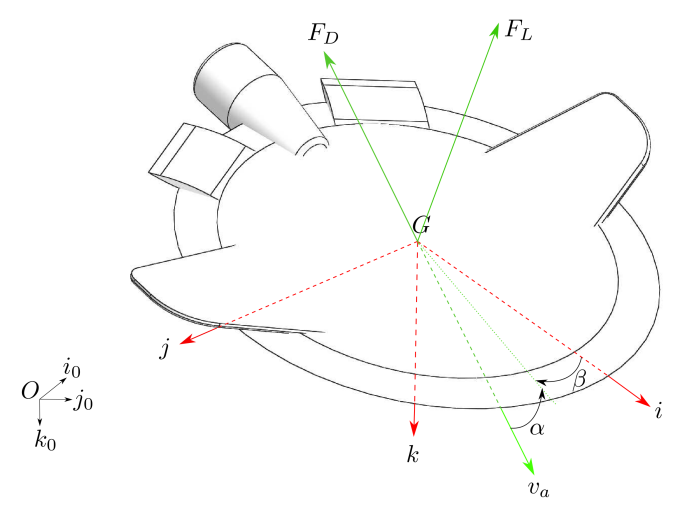

Fig. 1. Disc-shaped aircraft

To be usable for control design, the Newton equation (5) has to be complemented with a model of the resultant aerodynamic force $\boldsymbol{F}_{a}$. As explained previously we here consider an aircraft whose shape is approximated by a disc (see Fig. 1). We further assume that the plane $(G ; \boldsymbol{\imath}, \boldsymbol{\jmath})$ coincides with the disc surface. This is coherent with the fact that the propulsive force of an aircraft is approximately parallel to the plane's main wing and does not produce an important torque. From this assumption it also comes that $\imath$ (resp. ) is the longitudinal (resp. lateral) motion direction of the aircraft. For control design we propose to use the following model of $\boldsymbol{F}_{a}$, with $c_{0}$ and $c_{1}$ denoting two positive numbers, and $\bar{c}_{0}=c_{0}+2 c_{1}$ :

$$
\begin{aligned}
\boldsymbol{F}_{a} & =-c_{0}\left|\boldsymbol{v}_{a}\right| \boldsymbol{v}_{a}-2 c_{1}\left(\boldsymbol{v}_{a} \cdot \boldsymbol{k}\right)\left|\boldsymbol{v}_{a}\right| \boldsymbol{k} \\
& =\left(-\bar{c}_{0} \boldsymbol{v}_{a}+2 c_{1} v_{a, 1} \boldsymbol{\imath}+2 c_{1} v_{a, 2} \boldsymbol{\jmath}\right)\left|v_{a}\right|
\end{aligned}
$$

This model clearly respects the disc symmetries. Note that if the drag coefficient $c_{0}$ were equal to zero then $\boldsymbol{F}_{a}$ would be orthogonal to the disk plane with an amplitude proportional to $\sin \alpha\left|\boldsymbol{v}_{a}\right|^{2}$. This model is also compatible with the previous relations (3) and (4). Indeed, one easily verifies that, in this case, $\boldsymbol{v}_{a}^{\perp}=\frac{\left|v_{a}\right|}{\cos \alpha} \boldsymbol{k}-\tan \alpha \boldsymbol{v}_{a}$, $C_{D}(\alpha)=\left(c_{0}+2 c_{1} \sin ^{2} \alpha\right) / \eta_{a}$, and $C_{L}(\alpha)=c_{1} \sin 2 \alpha / \eta_{a}$. The symmetry of the disc w.r.t. its central axis also explains the non-dependence of the aerodynamic characteristics on the sideslip angle $\beta$. For small attack angles $|\alpha|$, the drag coefficient $C_{D}$ is thus approximately equal to $\frac{c_{0}}{\eta_{a}}$ and the lift coefficient $C_{L}$ is approximately proportional to the attack angle with the coefficient of proportionality given by $\frac{2 c_{1}}{\eta_{a}}$. This is coherent with experimental data performed on a variety of wing profiles and axisymmetric bodies [9]. However, for lift-optimized wing profiles this model fails to account for stall phenomena occurring at large attack angles. A way to tentatively overcome this shortcoming consists in changing the coefficients $c_{0}$ and $c_{1}$ beyond the stall angle.

\section{CONTROL DESIGN}

\section{A. Reference trajectory tracking}

Let $\boldsymbol{p}$ denote the disc CoM position, and $\boldsymbol{p}_{r}(t)$ a (three times differentiable) reference trajectory in $\boldsymbol{E}^{3}$ with bounded time-derivatives at all orders. The control objective is to stabilize the position tracking error $\tilde{\boldsymbol{p}}:=\boldsymbol{p}-\boldsymbol{p}_{r}$ and the sideslip angle (and thus $v_{a, 2}$ ) about zero. The latter objective corresponds to the achievement of a so-called balanced, or coordinated, flight that every pilot is instructed to perform in nominal flight conditions, for the comfort of the passengers (zero lateral acceleration) and to minimize drag. The feedback control laws derived thereafter potentially apply to any reference trajectory $\boldsymbol{p}_{r}(t)$. However, the accompanying stability analysis imposes to reduce the set of trajectories for which exponential convergence can be proved. This leads us to define a set of admissible trajectories for the considered aircraft as follows, with $\boldsymbol{v}_{r}(t)$ and $\boldsymbol{a}_{r}(t)$ denoting the first and second time-derivatives of $\boldsymbol{p}_{r}(t)$ respectively:

Definition 1 A trajectory $\boldsymbol{p}_{r}(t)$ such that $0<\epsilon<\left|v_{r}(t)\right|<$ $v_{\max }<+\infty$ is admissible if the aircraft equations (1)-(5), assuming zero wind velocity, are satisfied with

1) zero sideslip velocity, i.e. $\forall t: v_{r, 2}(t)=0$,

2) strictly positive (or negative) angles of attack, i.e. $\exists \epsilon_{1}>0, \forall t:\left|v_{r, 3}(t)\right|>\epsilon_{1}$,

3) the inequality:

$$
\exists \epsilon_{2}>0, \forall t:\left|\boldsymbol{g}-\frac{\bar{c}_{0}}{m}\right| v_{r}(t)\left|\boldsymbol{v}_{\boldsymbol{r}}(t)-\boldsymbol{a}_{\boldsymbol{r}}(t)\right|>\epsilon_{2}
$$

It is not difficult to verify that this set of trajectories is much larger than the set of classically defined trim trajectories for which the aircraft translational and angular velocities expressed in body frame are constant. The importance of the inequality (8) is that, combined with zero sideslip velocity and the inequality constraint upon the attack angle, it ensures the existence of only four possible aircraft orientations $\left(\boldsymbol{v}_{r}, \boldsymbol{J}_{r}, \boldsymbol{k}_{r}\right)(t)$ along the reference trajectory. More precisely, assuming zero wind velocity, i.e. $\boldsymbol{v}_{w}=0$, and defining $\boldsymbol{F}_{r}(t):=\frac{\bar{c}_{0}}{m}\left|v_{r}(t)\right| \boldsymbol{v}_{\boldsymbol{r}}(t)+\boldsymbol{a}_{\boldsymbol{r}}(t)-\boldsymbol{g}$, using the expression (7) of $\boldsymbol{F}_{a}$ in (5), and setting $\boldsymbol{v}_{r} \cdot \boldsymbol{J}_{r}=0$, yields (with the time index omitted for readability)

$$
\boldsymbol{F}_{r}=\frac{2 c_{1}\left(\boldsymbol{v}_{r} \cdot \boldsymbol{\imath}_{r}\right)\left|\boldsymbol{v}_{r}\right|+T}{m} \boldsymbol{\imath}_{r}
$$

This equation has two solutions, namely $\boldsymbol{\imath}_{r}= \pm \frac{\boldsymbol{F}_{r}}{\left|\boldsymbol{F}_{r}\right|}$ with $T=\left(\left(m \boldsymbol{F}_{r}-2 c_{1}\left|v_{r}\right| \boldsymbol{v}_{r}\right) \boldsymbol{\imath}_{r}\right)=\left(c_{0}\left|v_{r}\right| \boldsymbol{v}_{r}+m\left(\boldsymbol{a}_{r}-\boldsymbol{g}\right)\right) \boldsymbol{\imath}_{r}$. 
As for the unit vector $\boldsymbol{J}_{r}$ it has to be orthogonal to both $\boldsymbol{\imath}_{r}$ and $\boldsymbol{v}_{r}$ (so that $v_{r, 2}=\boldsymbol{v}_{r} \cdot \boldsymbol{J}_{r}=0$ ). Therefore $\boldsymbol{J}_{r}(t)=$ $\pm \frac{\boldsymbol{v}_{r}(t) \times \boldsymbol{\imath}_{r}(t)}{\left|\boldsymbol{v}_{r}(t) \times \boldsymbol{\imath}_{r}(t)\right|}$. Finally, the third unit vector $\boldsymbol{k}_{r}$ is just the cross-product of the other two unit vectors, i.e. $\boldsymbol{k}_{r}(t)=$ $\boldsymbol{\imath}_{r}(t) \times \boldsymbol{J}_{r}(t)$. These four orientations can be deduced from each other via a rotation of angle $\pi$. They arise from the disc symmetries and yield the same body profile in the air. Transposing them to the case of common aeroplane flying horizontally along a straight path and with a constant air velocity they correspond to flying either cockpit/up or cockpit/down with the aircraft nose or its tail facing the incoming air. Assuming positive coordinates along $\boldsymbol{\imath}_{r}$ and $\boldsymbol{k}_{r}$ for the cockpit position, the common situation (cockpit/up and nose facing the incoming air) corresponds to choosing $\boldsymbol{\imath}_{r}=\frac{\boldsymbol{F}_{r}}{\left|\boldsymbol{F}_{r}\right|}$ and $\boldsymbol{j}_{r}=\frac{\boldsymbol{v}_{r}(t) \times \boldsymbol{\imath}_{r}(t)}{\left|\boldsymbol{v}_{r}(t) \times \boldsymbol{\imath}_{r}(t)\right|}$. The thrust and the attack angles are then both positive. From now on we assume that this is the desired aircraft orientation along an admissible trajectory.

Let us now focus on control design. For the sake of simplification it is thereafter assumed that there is no wind so that $\boldsymbol{v}_{w}=0$ and $\boldsymbol{v}_{a}=\boldsymbol{v}$. We denote the acceleration tracking error as $\tilde{\boldsymbol{a}}=\boldsymbol{a}-\boldsymbol{a}_{r}$. Using the expression (7) of $\boldsymbol{F}_{a}$ in (5) then yields

$$
\tilde{\boldsymbol{a}}=\left(\boldsymbol{g}-\frac{\bar{c}_{0}}{m}|\boldsymbol{v}| \boldsymbol{v}-\boldsymbol{a}_{r}\right)+\frac{2 c_{1} v_{1}|\boldsymbol{v}|+T}{m} \boldsymbol{\imath}+\frac{2 c_{1} v_{2}|\boldsymbol{v}|}{m} \boldsymbol{\jmath}
$$

Let $\boldsymbol{I}_{\tilde{p}}$ denote a saturated integral of the position tracking error $\tilde{\boldsymbol{p}}$, and $\boldsymbol{\xi}\left(\tilde{\boldsymbol{p}}, \tilde{\boldsymbol{v}}, \boldsymbol{I}_{\tilde{p}}\right)$ denote a bounded PID-like control law that asymptotically (and locally exponentially) stabilizes $\left(\tilde{\boldsymbol{p}}, \tilde{\boldsymbol{v}}, \boldsymbol{I}_{\tilde{p}}\right)=(0,0,0)$ for the linear control system $\tilde{\boldsymbol{a}}=\boldsymbol{\xi}$, and such that $\boldsymbol{\xi}(0,0,0)=0$. In view of (9):

$$
\tilde{\boldsymbol{a}}=\boldsymbol{\xi}\left(\tilde{\boldsymbol{p}}, \tilde{\boldsymbol{v}}, \boldsymbol{I}_{\tilde{p}}\right)-\overline{\boldsymbol{F}}+\frac{\bar{T}}{m} \boldsymbol{\imath}+\frac{2 c_{1} v_{2}|\boldsymbol{v}|}{m} \boldsymbol{\jmath}
$$

with

$$
\begin{gathered}
\overline{\boldsymbol{F}}:=\frac{\bar{c}_{0}}{m}|\boldsymbol{v}| \boldsymbol{v}+\boldsymbol{a}_{r}-\boldsymbol{g}+\boldsymbol{\xi}\left(\tilde{\boldsymbol{p}}, \tilde{\boldsymbol{v}}, \boldsymbol{I}_{\tilde{p}}\right) \\
\bar{T}:=2 c_{1} v_{1}|\boldsymbol{v}|+T
\end{gathered}
$$

Set $\bar{T}=m(\overline{\boldsymbol{F}} . \boldsymbol{\imath})$ so that, in view of the previous relation, the thrust is calculated according to

$$
T=m(\overline{\boldsymbol{F}} . \boldsymbol{\imath})-2 c_{1} v_{1}|\boldsymbol{v}|
$$

Let us assume that $|\overline{\boldsymbol{F}}|$ is always larger than some positive number. Then the boundedness of $\left|\boldsymbol{a}_{r}-\boldsymbol{g}+\boldsymbol{\xi}\left(\tilde{\boldsymbol{p}}, \tilde{\boldsymbol{v}}, \boldsymbol{I}_{\tilde{p}}\right)\right|$ implies that there exists a positive number $\mu_{1}$ such that $|v|^{2}<\mu_{1}|\overline{\boldsymbol{F}}|$. Define the unit Euclidean vectors $\overline{\boldsymbol{\imath}}:=\frac{\overline{\boldsymbol{F}}}{|\overline{\boldsymbol{F}}|}$ and $\bar{\jmath}:=\frac{\boldsymbol{v} \times \overline{\boldsymbol{\imath}}}{|\boldsymbol{v} \times \overline{\boldsymbol{\imath}}|}$. Then (10) may also be written as

$$
\tilde{\boldsymbol{a}}=\boldsymbol{\xi}\left(\tilde{\boldsymbol{p}}, \tilde{\boldsymbol{v}}, \boldsymbol{I}_{\tilde{p}}\right)+|\overline{\boldsymbol{F}}|(\boldsymbol{\imath} \times(\boldsymbol{\imath} \times \overline{\boldsymbol{\imath}}))+\frac{2 c_{1} v_{2}|\boldsymbol{v}|}{m} \boldsymbol{\jmath}
$$

Now, from the fact that $v_{2}=\boldsymbol{v} \cdot \boldsymbol{\jmath}=\boldsymbol{v} \cdot(\boldsymbol{\jmath}-\overline{\boldsymbol{\jmath}})$, and since $|v|^{2}<\mu_{1}|\overline{\boldsymbol{F}}|$, there exists a positive number $\mu_{2}$ such that $\left|v_{2}\right||v|<\mu_{2}|\overline{\boldsymbol{F}}||\boldsymbol{\jmath}-\overline{\boldsymbol{\jmath}}|$. Therefore it suffices to work out an angular velocity control $\boldsymbol{\omega}$ that makes $|\overline{\boldsymbol{F}}|(|\boldsymbol{\imath}-\overline{\boldsymbol{\imath}}|+|\boldsymbol{\jmath}-\overline{\boldsymbol{\jmath}}|)$ converge to zero to obtain the closed-loop equation

$$
\tilde{\boldsymbol{a}}=\boldsymbol{\xi}\left(\tilde{\boldsymbol{p}}, \tilde{\boldsymbol{v}}, \boldsymbol{I}_{\tilde{p}}\right)+o(t), \lim _{t \rightarrow \infty} o(t)=0
$$

and ensure the convergence of $\left(\tilde{\boldsymbol{p}}, \tilde{\boldsymbol{v}}, \boldsymbol{I}_{\tilde{p}}\right)$ to $(0,0,0)$. This is the core of the control strategy that we propose. It implies in particular that the body frame $\mathcal{B}=\{G ; \boldsymbol{\imath}, \boldsymbol{J}, \boldsymbol{k}\}$ converges to the frame $\overline{\mathcal{B}}=\{G ; \overline{\boldsymbol{\imath}}, \overline{\boldsymbol{\jmath}}, \overline{\boldsymbol{k}}\}$ with $\overline{\boldsymbol{k}}:=\overline{\boldsymbol{\imath}} \times \overline{\boldsymbol{\jmath}}$. This latter problem is well posed because $\overline{\boldsymbol{F}}$ and $\boldsymbol{v}$ and, subsequently, the frame $\overline{\mathcal{B}}$, do not depend on (are not functions of) the disc orientation. This point is important to properly justify the proposed control design.

Define the angular velocities of $\overline{\boldsymbol{\imath}}$ and $\overline{\boldsymbol{j}}$ w.r.t. the inertial frame as $\boldsymbol{\omega}_{\overline{\boldsymbol{\imath}}}:=\overline{\boldsymbol{\imath}} \times \dot{\overline{\boldsymbol{\imath}}}$ and $\boldsymbol{\omega}_{\overline{\boldsymbol{\jmath}}}:=\overline{\boldsymbol{\jmath}} \times \dot{\overline{\boldsymbol{\jmath}}}$ respectively. Since $\overline{\boldsymbol{\imath}}$ and $\bar{\jmath}$ do not depend on the disc orientation, their time-derivatives do not depend on the disc angular velocity $\omega$. The angular velocity of the frame $\overline{\mathcal{B}}$ is then given by $\overline{\boldsymbol{\omega}}=\boldsymbol{\omega}_{\overline{\boldsymbol{\imath}}}+\left(\overline{\boldsymbol{\imath}} \cdot \boldsymbol{\omega}_{\bar{\jmath}}\right) \overline{\boldsymbol{\imath}}=$ $\boldsymbol{\omega}_{\bar{\jmath}}+\left(\bar{\jmath} \cdot \boldsymbol{\omega}_{\bar{\imath}}\right) \bar{\jmath}$, and this velocity does not depend on $\boldsymbol{\omega}$ either.

Lemma 1 Assume zero wind velocity and that the angle $|\tilde{\theta}|$ between the frames $\mathcal{B}$ at $\overline{\mathcal{B}}$ is initially smaller than $\pi$ (this maximal angle corresponds to a set of unstable equilibria). Provided that $|\overline{\boldsymbol{F}}|$ and $\left|\boldsymbol{v} \times \frac{\overline{\boldsymbol{F}}}{|\overline{\boldsymbol{F}}|}\right|$ are always larger than a small positive number so that $\overline{\boldsymbol{\imath}}$ and $\overline{\boldsymbol{\jmath}}$ are always well defined, the angular velocity control

$$
\boldsymbol{\omega}=\overline{\boldsymbol{\omega}}+\left(k_{\omega}(t)+\frac{\overline{\boldsymbol{F}} \cdot \dot{\overline{\boldsymbol{F}}}}{|\overline{\boldsymbol{F}}|^{2}+c}\right)((\boldsymbol{\imath} \times \overline{\boldsymbol{\imath}})+(\boldsymbol{\jmath} \times \overline{\boldsymbol{\jmath}})+(\boldsymbol{k} \times \overline{\boldsymbol{k}}))
$$

with $k_{\omega}(t)>\epsilon>0$ and $c$ any positive number, makes $|\overline{\boldsymbol{F}}|\left|\tan \left(\frac{\tilde{\theta}}{2}\right)\right|$ uniformly exponentially converge to zero and, subsequently, renders the equilibrium $\mathcal{B}=\overline{\mathcal{B}}$ exponentially stable.

If $|\overline{\boldsymbol{F}}|$ were constant, this would be a classical result. In the more advanced form here presented this result and its proof are simple generalizations of Proposition 3 stated in [9] where only the convergence of $\boldsymbol{\imath}$ to $\overline{\boldsymbol{\imath}}$ is needed. More precisely the proof involves the candidate Lyapunov function $\left.V=0.5\left(|\overline{\boldsymbol{F}}|^{2}+c\right) \tan ^{2}\left(\frac{\tilde{\theta}}{2}\right)\right)$ whose time-derivative is found equal to $\dot{V}=-2 k_{\omega}(t) V(\leq 0)$ when applying the control (15).

Remark: The velocity $|v|$ is ultimately uniformly upperbounded by virtue of the convergence of $\left(\tilde{\boldsymbol{p}}, \tilde{\boldsymbol{v}}, \boldsymbol{I}_{\tilde{p}}\right)$ to $(0,0,0)$. This in turn implies that $|\overline{\boldsymbol{F}}|,|T|,|\dot{v}|$ and $|\dot{\overline{\boldsymbol{F}}}|$ are also ultimately uniformly upperbounded. Therefore, by choosing $c$ as large as needed the term $\frac{|\overline{\boldsymbol{F}} \cdot \dot{\overline{\boldsymbol{F}}}|}{|\overline{\boldsymbol{F}}|^{2}+c}$ can be rendered arbitrarily small. This indicates that this term is technically needed to prove the result of Lemma 1 without assuming in advance that $|v|$ is bounded, but also that it is in practice of little importance and that it can be discarded in (15). The inefficiency of this term in practice also comes from that the thrust is always limited so that $|v|$ is automatically bounded by virtue of the system's passivity and energy dissipation associated with aerodynamic drag (the coefficient $c_{0}$ in the expression of $\boldsymbol{F}_{a}$ ).

Lemma 1 states conditions under which the angular velocity control (15) exponentially stabilizes $\{G ; \boldsymbol{\imath}, \boldsymbol{\jmath}, \boldsymbol{k}\}$ at $\{G ; \overline{\boldsymbol{\imath}}, \overline{\boldsymbol{j}}, \overline{\boldsymbol{k}}\}$ and makes $o(t)$ in relation (14) converge exponentially to zero, provided that the thrust control $T$ is chosen according to (13). Using the assumption that 
$\boldsymbol{\xi}\left(\tilde{\boldsymbol{p}}, \tilde{\boldsymbol{v}}, \boldsymbol{I}_{\tilde{p}}\right)$ asymptotically (and locally exponentially) stabilizes $\left(\tilde{\boldsymbol{p}}, \tilde{\boldsymbol{v}}, \boldsymbol{I}_{\tilde{p}}\right)=(0,0,0)$ for the linear control system $\tilde{\boldsymbol{a}}=\boldsymbol{\xi}$, one deduces that under the same conditions the control law (13)-(15) asymptotically (and locally exponentially) stabilizes $\left(\tilde{\boldsymbol{p}}, \tilde{\boldsymbol{v}}, \boldsymbol{I}_{\tilde{p}}, \mathcal{B}\right)=(0,0,0, \overline{\mathcal{B}})$. In the case where $\boldsymbol{p}_{r}(t)$ is an admissible trajectory, these conditions are, in view of the definition of such a trajectory, satisfied when $\xi\left(\tilde{\boldsymbol{p}}, \tilde{\boldsymbol{v}}, \boldsymbol{I}_{\tilde{p}}\right)=0$. Moreover $\overline{\mathcal{B}}$ then coincides with the frame $\overline{\mathcal{B}}_{r}=\left\{G ; \boldsymbol{\imath}_{r}, \boldsymbol{J}_{r}, \boldsymbol{k}_{r}\right\}$. This yields the following result

Theorem 1 Assuming zero wind velocity, if $\boldsymbol{p}_{r}(t)$ is an admissible trajectory, then the control law (13)-(15) locally exponentially stabilizes $\left(\tilde{\boldsymbol{p}}, \tilde{\boldsymbol{v}}, \boldsymbol{I}_{\tilde{p}}, \mathcal{B}\right)=\left(0,0,0, \overline{\mathcal{B}}_{r}\right)$.

\section{Remarks:}

1) The formal proof of this theorem, not reproduced here for lack of space, is a direct adaptation and extension of the proof of Proposition 4 in [9].

2) The conditions pointed out in Lemma 1 prevent us from stating a more global stability result. However, the practical stability domain can be quite large because the set where $|\overline{\boldsymbol{F}}|$ and $\left|\boldsymbol{v} \times \frac{\overline{\boldsymbol{F}}}{|\overline{\boldsymbol{F}}|}\right|$ are equal to zero is very "thin".

3) No condition has so far been put on the sign of the thrust intensity $T$, whereas only positive thrust can be produced for many aircraft. To take this limitation into account and obtain a result similar to Theorem 1 one only has to add it as a constraint in the definition of an admissible trajectory, i.e. by further requiring that $T_{r}=\left(c_{0}\left|v_{r}(t)\right| \boldsymbol{v}_{r}(t)+m\left(\boldsymbol{a}_{r}-\boldsymbol{g}\right)\right) ._{r}$ is always larger than some positive number.

\section{Simulations}

The object of this section is to illustrate the tracking performance of the control $(T, \boldsymbol{\omega})$ given by (13) and (15) applied to a modelled disk-shaped aircraft weighting $3 \mathrm{~kg}$ and whose wing radius is equal to 1.2 meters. The reference trajectory used for this simulation is periodic and given by

$$
\boldsymbol{p}_{r}(t)=a \sin (b t) \boldsymbol{\imath}_{0}+a \sin (2 b t) \boldsymbol{\jmath}_{0}+c \sin (4 b t) \boldsymbol{k}_{0}
$$

with $a=\frac{225}{\pi}, b=\frac{\pi}{15}$, and $c=10$. The projection of this trajectory on the horizontal plane is an eight-shaped Lissajous curve, and the vertical coordinate varies in the interval $[-10,+10]$ meters. The bounded correction $\boldsymbol{\xi}$ involved in $\overline{\boldsymbol{F}}$ is calculated according to

$$
\begin{aligned}
& \boldsymbol{\xi}\left(\tilde{\boldsymbol{p}}, \tilde{\boldsymbol{v}}, \boldsymbol{I}_{\tilde{\boldsymbol{p}}}\right)=-k_{p} s a t^{20}\left(\tilde{\boldsymbol{p}}+k_{i} \boldsymbol{I}_{\tilde{\boldsymbol{p}}}\right)-k_{v} s a t^{20}\left(\tilde{\boldsymbol{v}}+k_{i} \dot{\boldsymbol{I}}_{\tilde{\boldsymbol{p}}}\right) \\
& \dot{\boldsymbol{I}}_{\tilde{\boldsymbol{p}}}=k_{I}\left(-\boldsymbol{I}_{\tilde{\boldsymbol{p}}}+\operatorname{sat}^{10}\left(\boldsymbol{I}_{\tilde{\boldsymbol{p}}}+\tilde{\boldsymbol{p}} / k_{I}\right)\right)
\end{aligned}
$$

with $\operatorname{sat}^{\Delta}(x):=\inf (1, \Delta /|x|) x$ the classical saturation function, $k_{v}=3, k_{p}=k_{v}^{2} / 4, k_{i}=k_{v} / 4$, and $k_{I}=20$. The gain $k_{\omega}(t)$ involved in the expression (15) of $\boldsymbol{\omega}$ is here chosen constant and equal to 10 . Initial position and velocity conditions are $\boldsymbol{p}(0)=50 \boldsymbol{\imath}_{0}-50 \boldsymbol{k}_{0}$ (the initial distance between the aircraft and the reference trajectory is thus approximately equal to 70 meters) and $\boldsymbol{v}(0)=15 \boldsymbol{J}_{0}$. The initial aircraft orientation is $(\boldsymbol{\imath}, \boldsymbol{\jmath}, \boldsymbol{k})(0)=\left(\boldsymbol{\jmath}_{0},-\imath_{0}, \boldsymbol{k}_{0}\right)$.
In order to test the robustness of the control against modelling errors the aircraft parameters $\left(m=3, c_{0}=\right.$ $\left.0.009, c_{1}=1.35\right)$ are replaced by the approximated values ( $\hat{m}=2.7, \hat{c}_{0}=0.0135, \hat{c}_{1}=1.17$ ) for the control computation. Also, the thrust calculated with these values is applied to the aircraft with the multiplication factor 0.8. Furthermore the thrust intensity is saturated at $40 N$. Although these approximations prevent the tracking errors from converging exponentially to zero -as predicted by the theoretical analysis developed in the paper- the simulation results reported in Figs. 2-7 show that they mildly impair the overall control performance. In particular the norm of the position tracking error $\tilde{\boldsymbol{p}}$ (see Fig. 4) does not ultimately exceed 0.6 meter. Note also that the thrust saturation during the first ten seconds of flight does not prevent the rapid convergence of the aircraft towards the reference trajectory occurring after about 15 seconds.

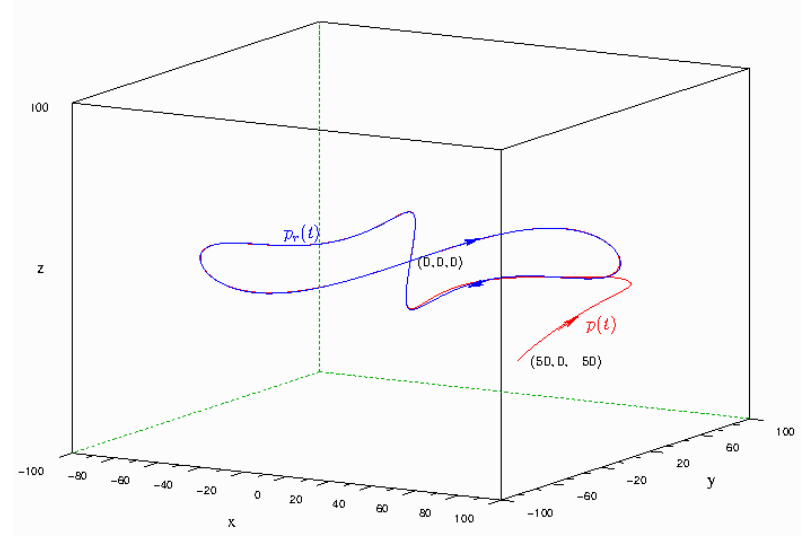

Fig. 2. Trajectories

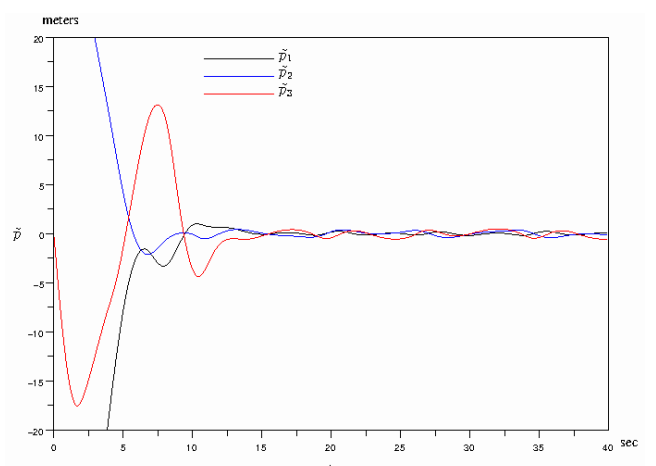

Fig. 3. Position tracking errors

\section{CONCLUDING REMARKS}

Nonlinear control of a disk-shaped scale-model aircraft has been addressed. The controller, designed on the basis of a generic model of aerodynamic forces acting on the vehicle, exponentially stabilizes any reference trajectory belonging to the large set of admissible trajectories that encompasses classical trim trajectories. This work is an extension of our 


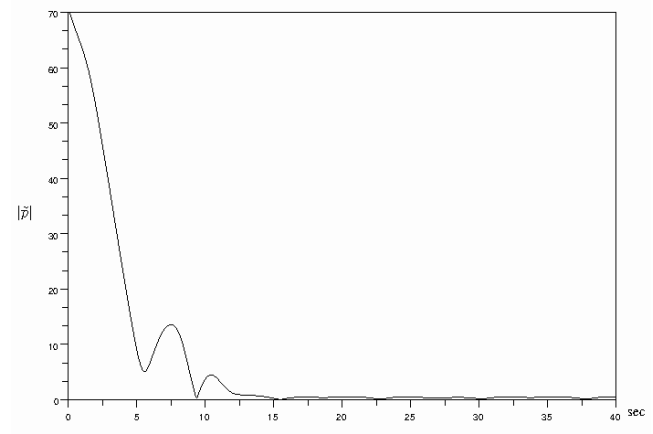

Fig. 4. Norm of position tracking errors

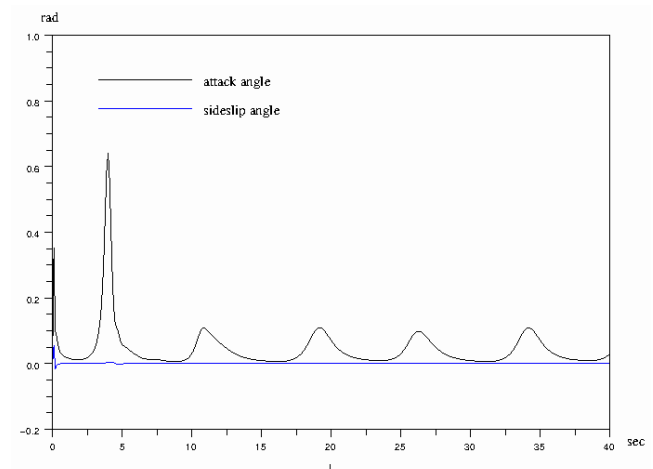

Fig. 5. Attack and sideslip angles

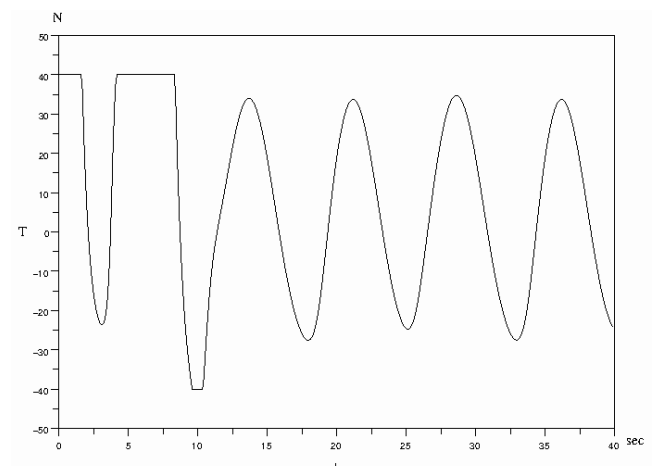

Fig. 6. Thrust control

prior works on the control of VTOL vehicles [2] and Missilelike vehicles [9], and it contributes to the development of a novel and unified control framework for aerial vehicles. We believe that the proposed control solution potentially applies to a large panel of scale-model airplanes with extended flight envelopes, but this claim remains to be consolidated by complementary investigations and results. Among many extensions that we envision let us cite, for instance

- the adaptation of the trajectory-tracking control solution here reported to the path-following problem more commonly considered for flight control;

- the use of models of aerodynamic forces representing more accurately the aerodynamics, including stall phe-

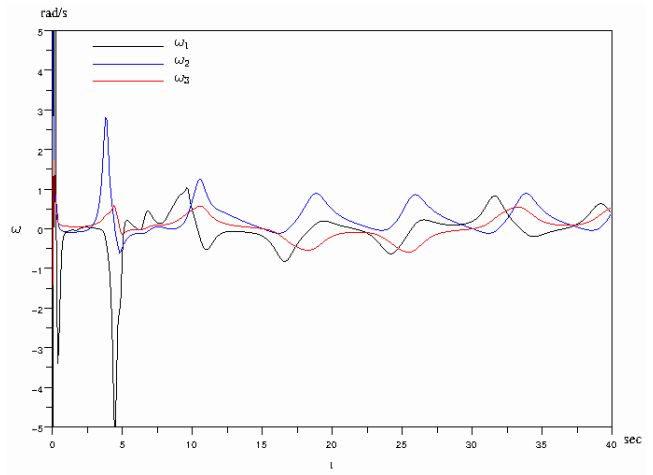

Fig. 7. Angular velocity control

nomona, of a typical airplane (with tail wings, dihedra, winglets,...), for both control design and simulation purposes;

- the adaptation of the control methodology to underactuated aircraft, i.e. with no tail-rudder for yaw control or no wing-ailerons for roll control.

- degraded versions of the control law in the case of partial velocity and orientation measurements, and in relation to the availability and use of existing miniaturized low-cost sensors, by taking advantage of aircraft passive stabilization properties.

\section{ACKNOWLEDGEMENT}

This research was partly supported by the French Agence Nationale de la Recherche via the ROBOTEX (ANR-10EQPX-44) project.

\section{REFERENCES}

[1] B. L. Stevens and F. L. Lewis, Aircraft control and simulation, ser Wiley-Interscience. New York, Chichester, Brisbane: J. Wiley and sons, 1992. [Online]. Available: http://opac.inria.fr/record=b1105347

[2] M.-D. Hua, T. Hamel, P. Morin, and C. Samson, "Introduction to feedback control of underactuated vtol vehicles: A review of basic control design ideas and principles," IEEE Control Systems, vol. 33, no. 1, pp. 61-75, 2013.

[3] L. Carter and J. Shamma, "Gain-scheduled bank-to-turn autopilot design using linear parameter varying transformations," Journal of Guidance, Control and Dynamics, vol. 27, no. 5, pp. 1056-1063, 1996.

[4] A. Marcos and G. Balas, "Development of linear parameter varying models for aircraft," Journal of Guidance, Control and Dynamics, vol. 27, pp. 218-228, 2004.

[5] G. D. G. Cai and C. Hu, "A velocity-based lpv modeling and control framework for airbreathing hypersonic vehicle," International Journal of Innovative Computing, Information and Control ICIC International, vol. 7, no. 5, pp. 2269-2281, 2011.

[6] B. Escande, Nonlinear dynamic inversion and $L Q$ techniques, ser. In: Magni JF., Bennani S., Terlouw J. (eds) Robust Flight Control, Lecture Notes in Control and Information Sciences, vol 224. Berlin, Heidelberg: Springer, 1997.

[7] Q. Wang and R. F. Stengel, "Robust nonlinear flight control of a high-performance aircraft," IEEE Transactions on Control Systems Technology, vol. 13, no. 1, pp. 15-26, 2005.

[8] M.-D. Hua, D. Pucci, T. Hamel, P. Morin, and C. Samson, "A novel approach to the automatic control of scale model airplanes," in in Proc. of Decision and Control Conference (CDC), 2014.

[9] D. Pucci, T. Hamel, P. Morin, and C. Samson, "Nonlinear feedback control of axisymmetric aerial vehicles," Automatica, vol. 53, pp. 72 $78,2015$. 